Article

\title{
Partial Linear NMF-Based Unmixing Methods for Detection and Area Estimation of Photovoltaic Panels in Urban Hyperspectral Remote Sensing Data †
}

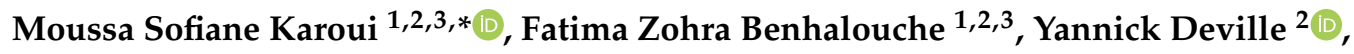 \\ Khelifa Djerriri ${ }^{1}$, Xavier Briottet ${ }^{4}(\mathbb{D})$, Thomas Houet ${ }^{5}{ }^{(D)}$, Arnaud Le Bris ${ }^{6}$ and \\ Christiane Weber ${ }^{7}$ (D) \\ 1 Centre des Techniques Spatiales, Arzew 31200, Algeria; Fatima.Benhalouche@irap.omp.eu (F.Z.B.); \\ kdjerriri@cts.asal.dz (K.D.) \\ 2 IRAP, Université de Toulouse, UPS, CNRS, CNES, 31400 Toulouse, France; Yannick.Deville@irap.omp.eu \\ 3 Université des Sciences et de la Technologie d'Oran Mohamed Boudiaf, Bir El Djir 31000, Oran, Algeria \\ 4 ONERA/DOTA Université de Toulouse, F-31055 Toulouse, France; Xavier.Briottet@onera.fr \\ 5 CNRS, Université Rennes 2, Unité Mixte de Recherche 6554 LETG, Place du Recteur Henri le Moal, \\ 35043 Rennes CEDEX, France; thomas.houet@univ-rennes2.fr \\ 6 Univ. Paris-Est, LASTIG STRUDEL, GN, ENSG, F-94160 Saint-Mandé, France; Arnaud.Le-Bris@ign.fr \\ 7 TETIS, CNRS, Univ. de Montpellier, F-34000 Montpellier, France; christiane.weber@teledetection.fr \\ * Correspondence: Sofiane.Karoui@irap.omp.eu \\ $+\quad$ This paper constitutes a substantial extension of: https://doi.org/10.1109/IGARSS.2018.8518204.
}

Received: 30 July 2019; Accepted: 13 September 2019; Published: 17 September 2019

\begin{abstract}
High-spectral-resolution hyperspectral data are acquired by sensors that gather images from hundreds of narrow and contiguous bands of the electromagnetic spectrum. These data offer unique opportunities for characterization and precise land surface recognition in urban areas. So far, few studies have been conducted with these data to automatically detect and estimate areas of photovoltaic panels, which currently constitute an important part of renewable energy systems in urban areas of developed countries. In this paper, two hyperspectral-unmixing-based methods are proposed to detect and to estimate surfaces of photovoltaic panels. These approaches, related to linear spectral unmixing (LSU) techniques, are based on new nonnegative matrix factorization (NMF) algorithms that exploit known panel spectra, which makes them partial NMF methods. The first approach, called Grd-Part-NMF, is a gradient-based method, whereas the second one, called Multi-Part-NMF, uses multiplicative update rules. To evaluate the performance of these approaches, experiments are conducted on realistic synthetic and real airborne hyperspectral data acquired over an urban region. For the synthetic data, obtained results show that the proposed methods yield much better overall performance than NMF-unmixing-based methods from the literature. For the real data, the obtained detection and area estimation results are first confirmed by using very high-spatial-resolution ortho-images of the same regions. These results are also compared with those obtained by standard NMF-unmixing-based methods and by a one-class-classification-based approach. This comparison shows that the proposed approaches are superior to those considered from the literature.
\end{abstract}

Keywords: hyperspectral imaging; hyperspectral unmixing; partial nonnegative matrix factorization; detection and area estimation; photovoltaic panels; urban areas 


\section{Introduction}

Operating renewable energy systems have been constantly and rapidly growing in recent years, mainly in urban areas of developed countries [1-3]. Photovoltaic panels, which are expected to offer a way to generate greener electrical power from non-polluting solar resources, represent an important component of these renewable energy systems. Their installations are part of urban energy strategic plans that have been highlighted by government agencies, electricity grid operators and decision makers to promote their installation and use through financial support and tax reduction. For that reason, and to avoid spiteful frauds with these substitute energies, several organizations are becoming ever more attracted to detailed information concerning these solar systems, as well as their localization and energy production [1].

Field surveys constitute one of the ways of obtaining such precise information, at least with regard to localization, on photovoltaic panels. However, this approach is not always effective or appropriate, since it consumes a lot of time and can be very expensive. Therefore, using other less expensive and faster methods is necessary.

The use of remote sensing data is an interesting approach to automatically detecting photovoltaic installations. In this context, some remote sensing-based approaches have recently been proposed $[1,4]$. These approaches exploit very high-spatial-resolution airborne/spaceborne RGB images with a small number of spectral bands. However, this type of data does not permit the efficient detection of solar panels, principally owing to their material properties. In fact, specular reflections, in some irradiation configurations, can alter the visual properties of these panels, and therefore their detection becomes difficult.

High-spectral-resolution airborne/spaceborne hyperspectral data represent an interesting option for surmounting the above-mentioned restrictions. Indeed, these remote sensing data are acquired by hyperspectral imaging sensors that collect data in hundreds of narrow and contiguous spectral bands, which permits more accurate material detection [5]. In particular, the basic idea when considering such remote sensing data for detecting and identifying a land surface material, including solar panels, consists of comparing, by using an appropriate criterion, each observed pixel-spectrum in an image with a reference one of the considered material [6]. Nevertheless, and mainly owing to the low spatial resolution of these sensors, mixed pixels, which contain several pure materials (also called endmembers), and therefore are characterized by mixtures of these endmember spectra, may exist in the collected images and then prevent direct detection of pure materials without sophisticated techniques to unmix these observed pixels.

To date, no hyperspectral-unmixing-based works have been reported in the literature for automatically detecting and estimating the surfaces of photovoltaic panels, other than the recent one proposed in [7]. In that work, a linear spectral unmixing (LSU) method was proposed to partially unmix a hyperspectral image, by means of a nonnegative matrix factorization (NMF) algorithm that exploits known solar panel spectra in order to automatically detect and estimate areas of this urban land surface material.

It should be noted that LSU methods, which can be viewed as typical linear blind source separation (BSS) approaches [8,9], are the most employed methods for processing hyperspectral remote sensing images. This process aims at linearly unmixing all observed spectra into a set of pure material spectra, and a collection of associated abundance fractions [10]. More specifically, NMF represents one of the most used techniques for performing the LSU process of remote sensing images. It consists of linearly decomposing a nonnegative matrix into a product of two nonnegative matrices [11]. Moreover, it must here be mentioned that LSU methods are generally considered when the landscape of the observed scene is flat and the irradiance is homogeneous. Otherwise, i.e., when the landscape is significantly non-flat and/or the irradiance is heterogeneous, especially in urban areas, LSU methods are not effective and must be replaced by nonlinear ones [12], in particular, those based on linear quadratic or bilinear mixing models [13-15]. In such cases, linear quadratic or bilinear matrix factorization methods, with nonnegativity constraints, may be considered [15-18]. 
The investigations reported in the present paper extend the above-mentioned approach of [7], still in a linear context. Indeed, in this paper, and in addition to the method described in [7], a new linear hyperspectral-unmixing method is designed for automatically detecting photovoltaic panels and estimating their surfaces. Thus, two linear methods are reported in this paper (and their experimental performance is analyzed in a much more detailed way than in [7]). These approaches are based on original algorithms that have relationships with NMF and that exploit known solar panel spectra. Therefore, the designed linear methods can be considered to be Partial (or informed) NMF (Part-NMF) techniques $[19,20]$. The first designed approach, called Gradient-based Part-NMF (Grd-Part-NMF), employs a projected gradient descent algorithm by means of iterative update rules that include the nonnegativity constraint, whereas the second proposed one [7], called Multiplicative Part-NMF (Multi-Part-NMF), uses multiplicative and iterative gradient-based update rules.

To evaluate the performance of the proposed approaches, experiments, based on realistic synthetic and real airborne hyperspectral data acquired over an urban region, are conducted. The obtained results are compared to those obtained by NMF-unmixing-based methods in the literature for synthetic and real data, and also to those obtained using a one-class-classification-based approach for the real data. Additionally, and still for the real data, obtained detection and area estimation results are confirmed by using ortho-images, of the same regions, with very high spatial resolution.

The remainder of this paper is structured in the following manner. The next section defines the mathematical data model used in the proposed approaches. The proposed algorithms are presented in Section 3. In Section 4, the realistic synthetic and real data employed are described. Section 5 consists of test results with the considered data. In Section 6, a discussion of the obtained results is given. Finally, conclusions are provided in the last section.

\section{Data Model}

As introduced above, the aim of the designed methods in these investigations is to automatically detect solar panels and to estimate their surfaces from an observed high-spectral-resolution hyperspectral remote sensing image $X \in R_{+}^{N \times K}$. $N$ and $K$ correspond, respectively, to the numbers of spectral bands and pixels of the considered hyperspectral image.

As mentioned in the previous section, the designed methods are related to LSU techniques, in which each observed nonnegative reflectance spectrum related to a pixel in an image is considered to be a linear mixture of the nonnegative reflectance endmember spectra inside the same pixel [10]. Thus, the whole observed nonnegative reflectance image $X$ can be modeled, in matrix form, as [21-23].

$$
X=A S
$$

Each row of the above matrix $X$ is one spectral band of the considered image. The $K$ pixels of this image are reorganized as a one-dimensional array. Each column of the matrix $A \in R_{+}^{N \times L}$ is one nonnegative endmember spectrum and each row of the matrix $S \in R_{+}^{L \times K}$ corresponds to all nonnegative abundance fractions, in all pixels, of one endmember. These coefficients should respect the well-known abundance sum-to-one constraint [10]. $L$ corresponds to the number of endmembers.

Again, and as introduced in the previous section, the NMF-LSU-based proposed methods, in which some photovoltaic panel spectra are assumed to be known, are designed to automatically detect these solar systems in urban hyperspectral data and to estimate their surfaces. Consequently, the matrix $A$ can be expressed as

$$
A=A_{1}+A_{2}
$$

The first $L_{1}$ columns of the matrix $A_{1} \in R_{+}^{N \times L}$ correspond to the known photovoltaic panel spectra. The other columns of this first matrix are composed of zeros. The last $L_{2}$ columns of the second matrix $A_{2} \in R_{+}^{N \times L}$ correspond to the unknown endmember spectra. The other columns of this second matrix are composed of zeros. Evidently, $L=L_{1}+L_{2}$. Therefore, Equation (1) becomes 


$$
X=\left(A_{1}+A_{2}\right) S=A_{1} S+A_{2} S
$$

In this equation, the unknown matrices, which will be estimated by the designed algorithms, described in the next section, are $A_{2}$ and, especially, $S$, which contains photovoltaic panel abundance fractions, allowing the detection of these solar systems and the estimation of their areas.

\section{Proposed Approaches}

The designed iterative Part-NMF algorithms are similar to the literature NMF approaches that employ iterative multiplicative [24,25] or projected gradient [26] update rules to perform the NMF-unmixing process. In these methods, two nonnegative matrix variables are involved. These matrices are $\widetilde{A}_{2}$ and $\widetilde{S}$, which respectively aim at estimating $A_{2}$ and $S$, such that

$$
X \approx A_{1} \widetilde{S}+\widetilde{A_{2}} \widetilde{S}
$$

Accordingly, the designed approaches can be seen as partial/informed NMF ones [19,20]. These designed iterative algorithms consist in minimizing the following criterion

$$
J=\frac{1}{2}\left\|X-A_{1} \widetilde{S}-\widetilde{A_{2}} \widetilde{S}\right\|_{F}^{2}
$$

by means of iterative gradient-based update rules. $\|\cdot\|_{F}$ corresponds to the Frobenius norm.

Since the proposed methods are gradient-based ones, the criterion $J$ is rewritten as follows to easily obtain the gradient expressions

$$
J=\frac{1}{2} \operatorname{Tr}\left(X X^{\mathrm{T}}\right)-\operatorname{Tr}\left(A_{1} \widetilde{S} X^{\mathrm{T}}\right)-\operatorname{Tr}\left(\widetilde{A_{2}} \widetilde{S} X^{\mathrm{T}}\right)+\operatorname{Tr}\left(\widetilde{A_{2}} \widetilde{S S}^{\mathrm{T}} A_{1}^{\mathrm{T}}\right)+\frac{1}{2} \operatorname{Tr}\left(A_{1} \widetilde{S S}^{\mathrm{T}} A_{1}^{\mathrm{T}}\right)+\frac{1}{2} \operatorname{Tr}\left(\widetilde{A_{2}} \widetilde{S S}^{\mathrm{T}} \widetilde{A}_{2}^{\mathrm{T}}\right)
$$

where $\operatorname{Tr}($.$) and (.)^{\mathrm{T}}$, respectively, correspond to the matrix trace and the matrix transpose. With this expression and using the properties reported in [27], the gradient expressions of $J$, with respect to the two variables, are in matrix form

$$
\begin{gathered}
\frac{\partial J}{\partial \widetilde{A}_{2}}=-X \widetilde{S}^{\mathrm{T}}+A_{1} \widetilde{S S^{\mathrm{T}}}+\widetilde{A_{2}} \widetilde{S S}^{\mathrm{T}} \\
\frac{\partial J}{\partial \widetilde{S}}=-A_{1}^{\mathrm{T}} X-\widetilde{A}_{2}^{\mathrm{T}} X+\widetilde{A}_{2}^{\mathrm{T}} A_{1} \widetilde{S}+A_{1}^{\mathrm{T}} \widetilde{A_{2}} \widetilde{S}+A_{1}^{\mathrm{T}} A_{1} \widetilde{S}+\widetilde{A}_{2}^{\mathrm{T}} \widetilde{A_{2}} \widetilde{S}
\end{gathered}
$$

For the designed Grd-Part-NMF method, and using a generalized gradient descent algorithm, the following update rules are obtained:

$$
\begin{gathered}
\widetilde{A_{2}} \leftarrow \widetilde{A_{2}}-\varphi_{\widetilde{A}_{2}} \odot \frac{\partial J}{\partial \widetilde{A}_{2}} \\
\widetilde{S} \leftarrow \widetilde{S}-\varphi_{\widetilde{S}} \odot \frac{\partial J}{\partial \widetilde{S}}
\end{gathered}
$$

where $\odot$ represents the element-wise multiplication. $\varphi_{\widetilde{A}_{2}}$ and $\varphi_{\widetilde{S}}$ correspond to small positive and appropriately fixed or adaptive learning rates in matrix form (selected by means of the "Armijo rule along the projection arc" as reported in [26], and therefore this implies a higher computation time). These update rules do not guarantee nonnegativity, and therefore they are not sufficient. Thus, and to meet this constraint, the maximum, between each element of the updated matrix and a very small 
positive value $\varepsilon$ (usually set to the default MATLAB epsilon value), is kept [11]. Accordingly, the final gradient-based iterative update rules for the first proposed method read

$$
\begin{aligned}
\widetilde{A_{2}} & \leftarrow \max \left\{\widetilde{A_{2}}-\varphi_{\widetilde{A}_{2}} \odot \frac{\partial J}{\partial \widetilde{A}_{2}}, \varepsilon\right\} \\
\widetilde{S} & \leftarrow \max \left\{\widetilde{S}-\varphi_{\widetilde{S}} \odot \frac{\partial J}{\partial \widetilde{S}}, \varepsilon\right\}
\end{aligned}
$$

For the proposed Multi-Part-NMF method, multiplicative and iterative update rules are derived from the gradient-based update rules (9) and (10). These rules are obtained by formulating the learning rate matrices $\varphi$. as functions of the used matrices so as to obtain a multiplicative update and to preserve the nonnegativity constraint. Indeed, each gradient expression of $J$ with respect to $\theta$ (which corresponds to one of the variable matrices $\widetilde{A}_{2}$ or $\widetilde{S}$ ) can be written as the difference of two nonnegative functions such that

$$
\frac{\partial J}{\partial \theta}=\frac{\partial J}{\partial \theta}^{+}-\frac{\partial J}{\partial \theta}^{-}
$$

The function $\frac{\partial J}{\partial \theta}^{+}$is obtained by keeping the terms of (7) or (8) having a plus sign, whereas $\frac{\partial J}{\partial \theta}^{-}$is obtained by keeping the terms having a minus sign. The multiplicativity and nonnegativity constraints can be satisfied by initializing $\theta$ with a nonnegative value and choosing the value of the learning rate matrix $\varphi_{\theta}$ according to

$$
\varphi_{\theta}=\theta \oslash \frac{\partial J}{\partial \theta}^{+}
$$

where $\oslash$ denotes the element-wise division. Thus, the following learning rate matrices are obtained

$$
\begin{gathered}
\varphi_{\widetilde{A}_{2}}=\widetilde{A_{2}} \oslash\left(A_{1} \widetilde{S S}^{\mathrm{T}}+\widetilde{A_{2}} \widetilde{S S}^{\mathrm{T}}\right) \\
\varphi_{\widetilde{S}}=\widetilde{S} \oslash\left(\widetilde{A}_{2}^{\mathrm{T}} A_{1} \widetilde{S}+A_{1}^{\mathrm{T}} \widetilde{A_{2}} \widetilde{S}+A_{1}^{\mathrm{T}} A_{1} \widetilde{S}+\widetilde{A}_{2}^{\mathrm{T}} \widetilde{A_{2}} \widetilde{S}\right)
\end{gathered}
$$

Accordingly, and considering each update rule (9) and (10) takes the following form:

$$
\theta \leftarrow \theta \odot{\frac{\partial J^{-}}{\partial \theta}}^{\ominus}{\frac{\partial J^{+}}{\partial \theta}}^{+}
$$

the final designed multiplicative and iterative update rules for the $\widetilde{A}_{2}$ and $\widetilde{S}$ matrix variables are

$$
\begin{gathered}
\widetilde{A}_{2} \leftarrow \widetilde{A_{2}} \odot X \widetilde{S}^{\mathrm{T}} \oslash\left(A_{1} \widetilde{S S}^{\mathrm{T}}+\widetilde{A}_{2} \widetilde{S S}^{\mathrm{T}}+\epsilon\right) \\
\widetilde{S} \leftarrow \widetilde{S} \odot\left(A_{1}^{\mathrm{T}} X+\widetilde{A}_{2}^{\mathrm{T}} X\right) \oslash\left(\widetilde{A}_{2}^{\mathrm{T}} A_{1} \widetilde{S}+A_{1}^{\mathrm{T}} \widetilde{A_{2}} \widetilde{S}+A_{1}^{\mathrm{T}} A_{1} \widetilde{S}+\widetilde{A}_{2}^{\mathrm{T}} \widetilde{A_{2}} \widetilde{S}+\epsilon\right)
\end{gathered}
$$

where $\epsilon$ is a very small and positive value, again usually set to the default MATLAB epsilon value, which is added to the denominator of each multiplicative update rule to prevent possible division by zero.

The above-designed update rules (11) and (12) for the Grd-Part-NMF method or (18) and (19) for the Multi-Part-NMF method, in addition to guaranteeing the nonnegativity constraint (when the matrix variables are initialized with nonnegative values for the Multi-Part-NMF method), experimentally give a descent algorithm, so that $J$ decreases during iterations.

The proposed Part-NMF algorithms, similar to the literature NMF methods, have limitations: they are not guaranteed to offer a unique solution and their convergence point may depend on their initialization. In fact, the initialization stage of these algorithms is their key problem. To solve this issue, and to prevent random initialization from the point of view of the designed Part-NMF algorithms, and as the initialization step, initial estimates of unknown endmember spectra, stacked in the last $L_{2}$ columns of the matrix $\widetilde{A_{2}}$ may, e.g., be computed by the Vertex Component Analysis (VCA) [28] or the 
Simplex Identification via Split Augmented Lagrangian (SISAL) [29] methods, which are among the most famous approaches used in LSU techniques. The VCA and SISAL methods require the number $L$ of known and unknown pure materials to be known. It may be automatically determined by using the Hyperspectral Signal Subspace Identification by Minimum Error (HySime) method [30]. It should be noted that in the proposed methods, among the $L$ spectra estimated by the VCA or SISAL methods, the $L_{1}$ estimated spectra which are the most similar to the known ones, which correspond to photovoltaic panels, are replaced by those known for these solar systems in the set of $L$ estimated spectra. In addition, each initial value of the matrix $\widetilde{S}$ may be set to $1 / L$ or estimated, from the observed image $X$ and the known and initial estimated unknown endmember spectra, by using the Fully Constrained Least Squares (FCLS) method [31], separately applied to each pixel of the observed image.

The abundance sum-to-one property is an additional constraint to be considered during optimization. For this purpose, the approach mentioned in [31] is used throughout the optimization stage mentioned hereafter, in addition to the initialization mentioned above.

After initializing the two considered matrices as mentioned above, the optimization phase consists of repeatedly updating these matrices, until convergence (reported hereafter), according to (11) and (12) for the Grd-Part-NMF method or (18) and (19) for the Mult-Part-NMF method, and by considering the technique described in [31] to meet the abundance sum-to-one property.

Checking if the relative modification of the criterion $J$ takes a value below a predefined threshold thresh constitutes the first stopping criterion of the proposed Part-NMF algorithms, which reads

$$
\left|\frac{J^{|t|}-J^{|t+1|}}{J^{|t|}}\right| \leq \text { thresh }
$$

where $t$ corresponds to an iteration. The predefined threshold can be empirically chosen, after running the proposed methods with different threshold values, to obtain the most convincing results, but not necessarily to improve results with respect to most used performance evaluation criteria. Indeed, the choice of other values of the above thresh parameter may not influence the values of the performance criteria in some domains. In addition, and to prevent a high number of iterations, an additional stopping criterion is considered by checking if this number of iterations exceeds a predefined maximum.

The solar panels area is obtained, in each pixel, by multiplying the estimated abundance thereof by the pixel area of the considered image. The total solar panels area is obtained by aggregating all areas obtained in each pixel.

The complete algorithms of the proposed Part-NMF methods are described below. 
Algorithm 1: Grd-Part-NMF unmixing for automatically detecting photovoltaic panels and estimating their areas in urban hyperspectral remote sensing data.

Input: hyperspectral image $X$ and known photovoltaic panel spectra stacked in the first $L_{1}$ columns of the matrix $A_{1}$.

1. Estimate the number $L$ of known (photovoltaic panels) and unknown endmembers by means of the HySime Method.

2. Initialization stage

2.1. Initialize $\widetilde{A_{2}}$ from $X$ by means of the VCA or SISAL methods.

2.2. Set each element of $\widetilde{S}$ to $1 / L$ or initialize it from $X$ and known photovoltaic panel spectra and unknown endmember spectra by using the FCLS method.

3. Optimization stage (until convergence, for each iteration and considering the abundance sum-to-one property by using the technique described in [31])

3.1. Optimize $\widetilde{A_{2}}$ by using (11).

3.2. Optimize $\widetilde{S}$ by using (12).

Output: Photovoltaic panel abundance fractions, which allow their detection and their area estimation, and unknown spectra and their abundance fractions.

Algorithm 2: Multi-Part-NMF unmixing for automatically detecting photovoltaic panels and estimating their areas in urban hyperspectral remote sensing data.

Input: hyperspectral image $X$ and known photovoltaic panel spectra stacked in the first $L_{1}$ columns of the matrix $A_{1}$.

1. Estimate the number $L$ of known (photovoltaic panels) and unknown endmembers by means of the HySime Method.

2. Initialization stage

2.1. Initialize $\widetilde{A}_{2}$ from $X$ by means of the VCA or SISAL methods.

(a) Set each element of $\widetilde{S}$ to $1 / L$ or initialize it from $X$ and known photovoltaic panel spectra and unknown endmember spectra by using the FCLS method.

3. Optimization stage (until convergence, for each iteration and considering the abundance sum-to-one property by using the technique described in [31])

3.1. Optimize $\widetilde{A_{2}}$ by using (18).

3.2. Optimize $\widetilde{S}$ by using (19).

Output: Photovoltaic panel abundance fractions, which allow their detection and their area estimation, and unknown spectra and their abundance fractions.

Finally, it is worth emphasizing that the proposed approaches can be preceded by a preliminary and optional step that consists of selecting, from input data, zones that potentially contain photovoltaic panels. Thus, this step allows working only on small zones, and consequently it will lead to an acceleration of the detection and area estimation of the considered urban material. This step can be achieved by using a similarity parameter calculated between the reference spectrum of solar panels and each measured spectrum in the data. This will result in a similarity map from which it will be possible to select only the potential zones, around pixels with high similarity values, which contain the considered urban material. One of the most used similarity parameters is based on calculating the absolute value (|.|) of the correlation coefficient (CC) between the reference spectrum of solar panels and each measured spectrum. This parameter is defined as

$$
\mathrm{CC}=\frac{\left\langle r s_{p v}, m s\right\rangle}{\left\|r s_{p v}\right\| \cdot\|m s\|}
$$


where $r s_{p v}$ is the reference spectrum of photovoltaic panels and $m s$ corresponds to each measured spectrum. <., .> and $\|$.$\| , respectively, stand for the inner product and vector norm. Unlike the core of$ the proposed approaches, it is clear that this preliminary step is not sufficient in itself, since it does not allow calculating surfaces of the considered urban material with a sub-pixel accuracy.

\section{Materials}

Experiments considering realistic synthetic and real airborne hyperspectral data are conducted to assess the performance of the proposed algorithms.

\subsection{Realistic Synthetic Hyperspectral Data}

Four sets of urban environment spectra (photovoltaic panels, tiles, grass and trees: see Figure 1 , with spectral variability, are considered to generate, according to the linear mixing model (1), realistic synthetic hyperspectral data. To validate the robustness of the tested methods to spectral variability in the experiments carried out and described hereafter, one randomly selected spectrum, from each of these four sets, is used to create each pixel of the considered realistic synthetic image. The above pure spectra contain 214 samples (after removing the atmospheric absorption spectral bands and based on those considered in the real-airborne hyperspectral image described in the next subsection), ranging from 400 to $2500 \mathrm{~nm}$, and are obtained by ground measurements using a spectrometer. In all the conducted tests, for both realistic synthetic and real data, the mean spectrum (Figure 1) of the ground-measured solar panel spectra is considered to be the only known spectrum (i.e., $L_{1}=1$ ).

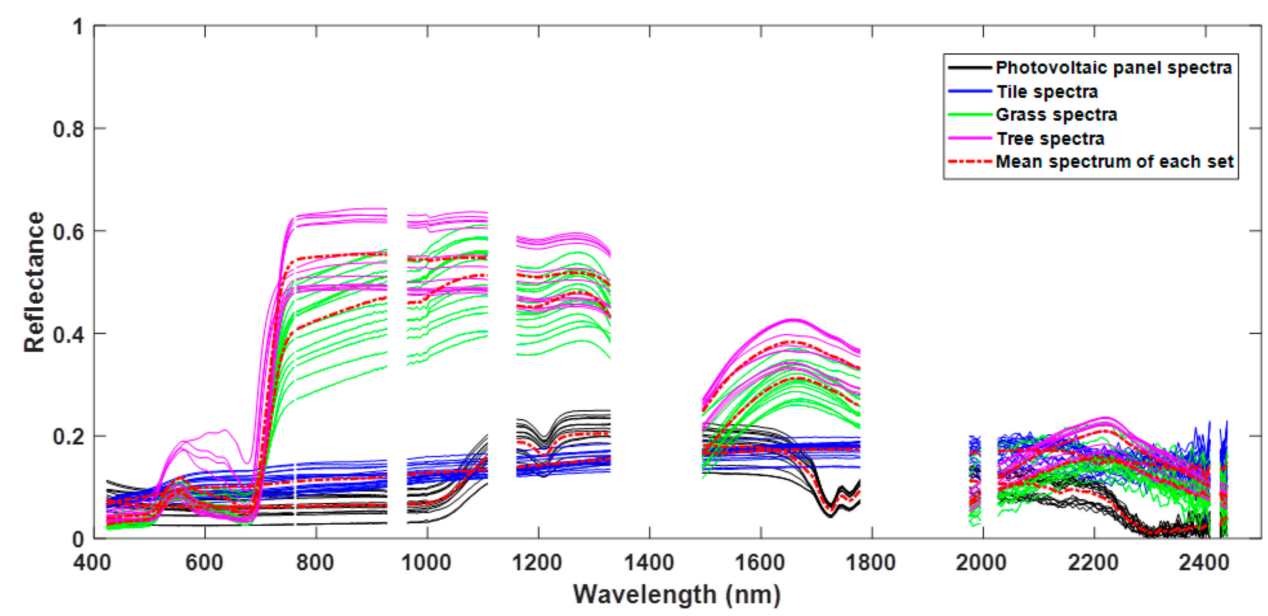

Figure 1. The four sets of urban environment spectra, and their means, used for creating the realistic synthetic hyperspectral data (the gaps correspond to the removed atmospheric absorption spectral bands).

The used abundance fraction maps (Figure 2c), with $10 \times 10$ pixels, are generated from a land cover classification (Figure $2 b$ ) of a real RGB urban image (Figure 2a) of $90 \times 90$ pixels, with the mentioned four materials, by averaging pixel classification values on a non-overlapping $9 \times 9$-pixel sliding window. This procedure allows the generation of synthetic abundance fractions in order to create realistic synthetic data. Moreover, it must be mentioned here that since the considered mixing model in these investigations is the standard linear mixing model, the edge phenomenon (which can appear when using sliding windows) does not occur. Indeed, and based on the used mixing model, each classified pixel, even being on the edge of one of the used non-overlapping and sliding windows, will have an effect only on the mixed pixel created from the classified pixels being in the same window, and thus will have no effect on the mixed pixels created from the adjacent windows. The edge phenomenon may appear, and must be taken into account, when the mixing model is more complex. 


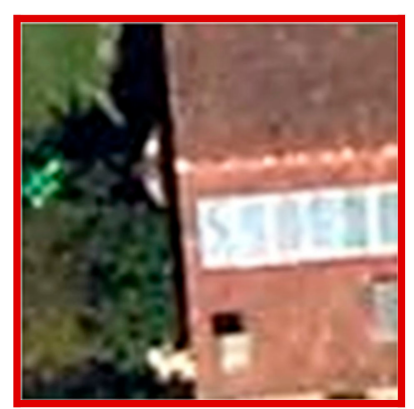

(a)

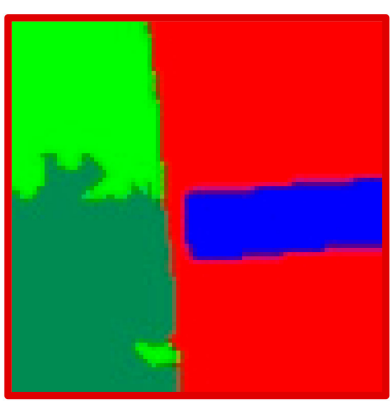

(b)

Figure 2. Cont.

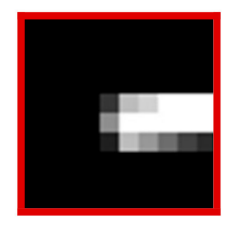

Photovoltaic panel

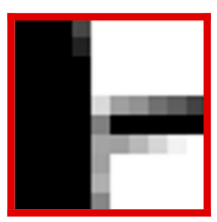

Tile

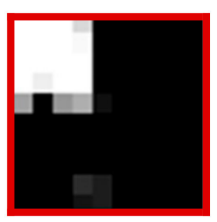

Grass

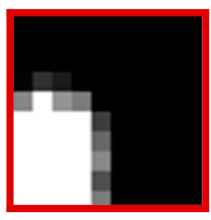

Tree

(c)

Figure 2. (a) The considered real urban image with four urban materials. (b) The land cover classification, with four classes: photovoltaic panel, tile, grass and tree, of the considered real urban image. (c) The four created abundance fraction maps.

\subsection{Real Airborne Hyperspectral Data}

As mentioned above, real airborne hyperspectral data are also considered in these investigations. The full used hyperspectral image covers a part of Toulouse (France) urban area (Figure 3). Toulouse downtown is typical from old cities situated in the South of France with a large number of components/characteristics [32,33]: tile roofs, low rise homes, big architectural monuments (cathedral, town hall, etc.), dense urbanization, small streets, large avenues and green spaces (public gardens, squares, etc.). Also, the suburbs are either residential private houses/buildings or large commercial/industrial buildings. This work focuses on the city center (Figure 4). 


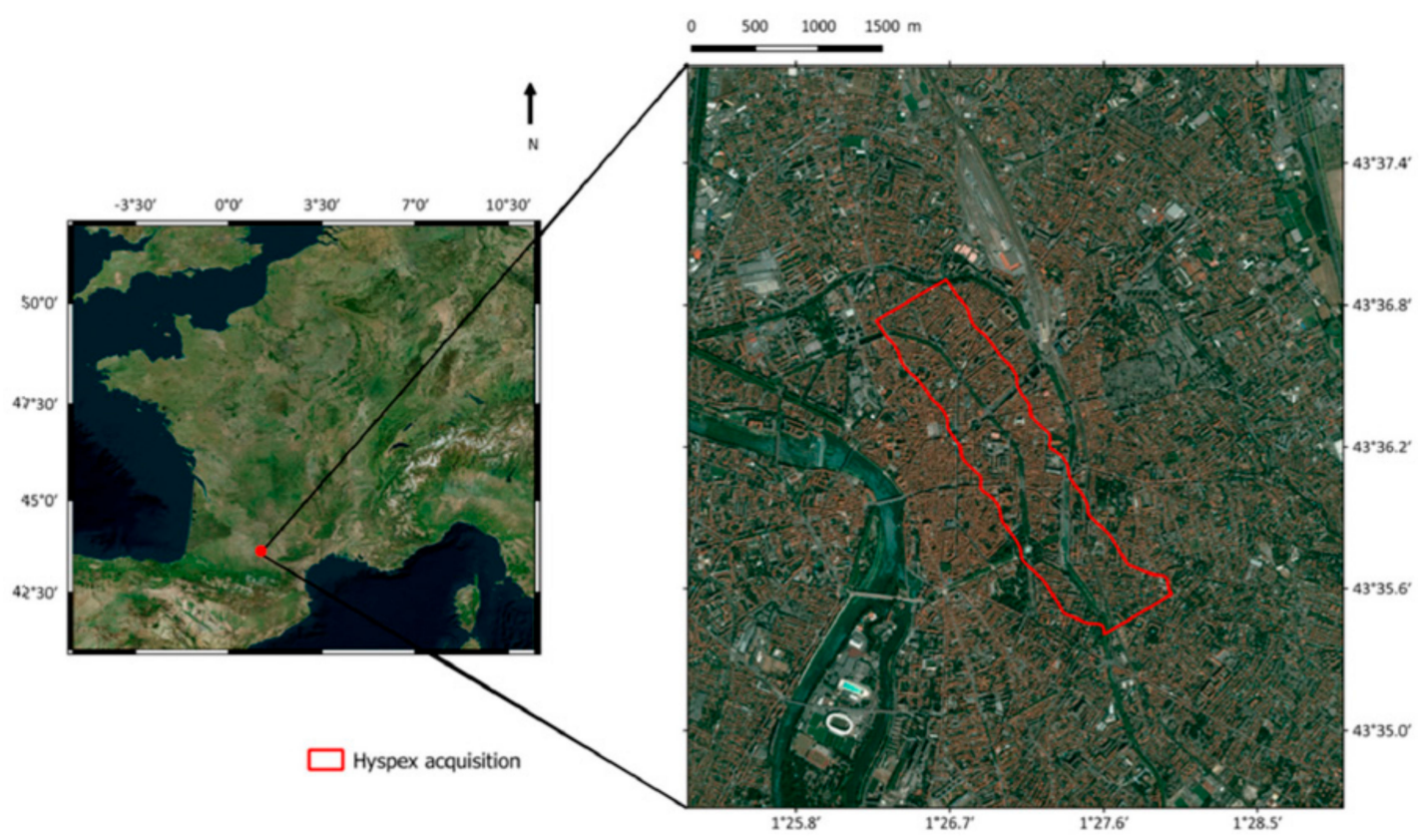

Figure 3. The considered Toulouse (France) urban area [33].

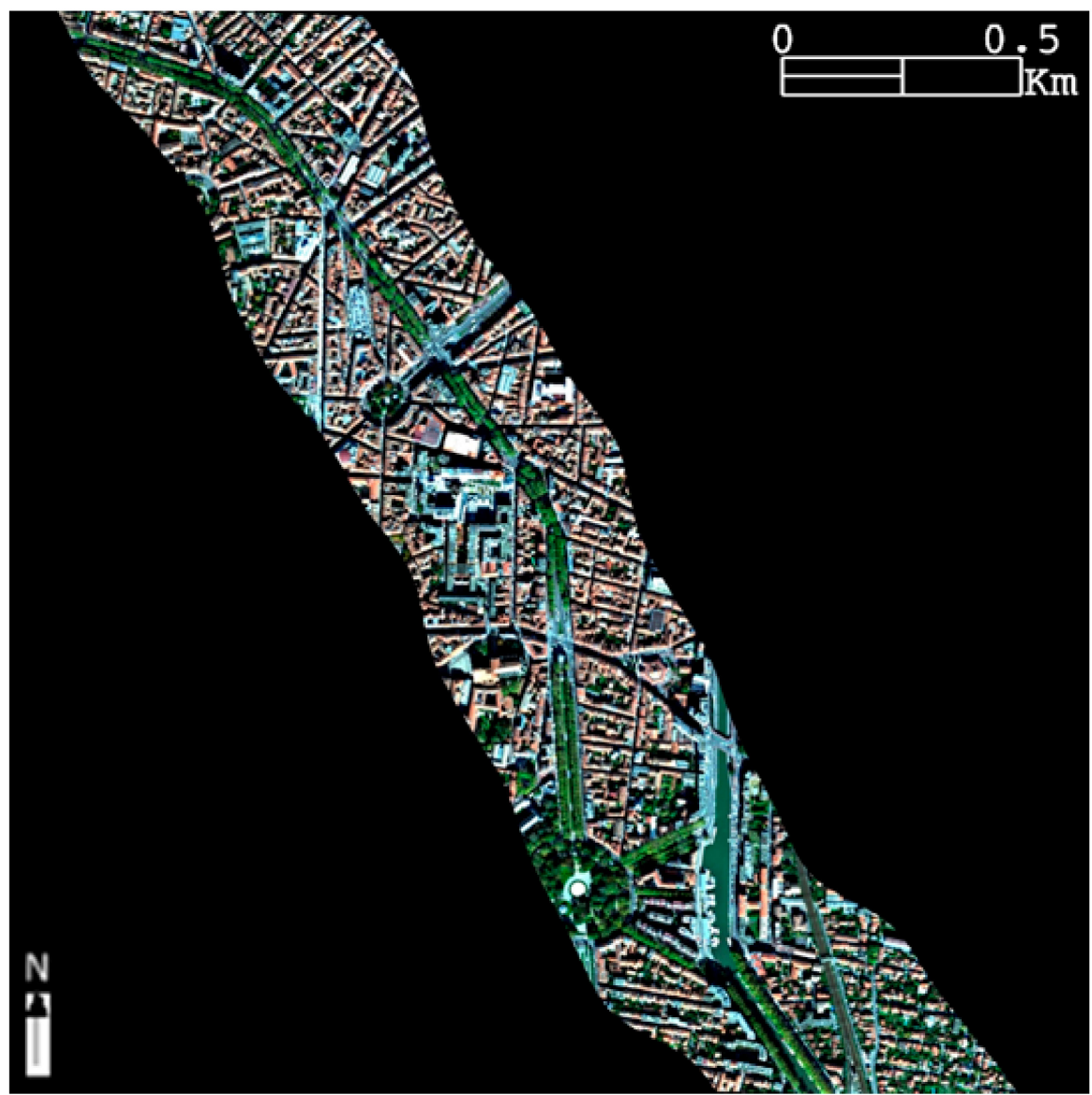

Figure 4. True color image composite of the considered full hyperspectral image.

The French airborne UMBRA (Urban Material characterization in the sun and shade of Built-up structures and trees and their Retrieval from Airborne image) campaign was conducted in October 2012 [34] by using two Hyspex hyperspectral cameras: the first one collects data in the Visible Near 
Infra-Red (VNIR) domain (400 to $1000 \mathrm{~nm}$ ) with $0.84 \mathrm{~m}$ spatial resolution and $3.7 \mathrm{~nm}$ spectral resolution, while the second sensor covers the Short Wave Infra-Red (SWIR) range (1000 to $2500 \mathrm{~nm}$ ), with $1.6 \mathrm{~m}$ spatial resolution and $6 \mathrm{~nm}$ spectral resolution. To work with the same frame of reference, the VNIR and SWIR images are co-registered, and the VNIR image is resampled to $1.6 \mathrm{~m}$ spatial resolution. The obtained airborne hyperspectral image contains 405 spectral bands over the range: 400 to $2500 \mathrm{~nm}$. An atmospheric correction is also applied to the considered image using the COrrection Code for Hyperspectral Images of remote sensing SEnsors (COCHISE) method [35]. The obtained pixel-spectra are reflectance ones. From the above-mentioned 405 spectral bands, only 214, still covering the $400 \mathrm{~nm}$ to $2500 \mathrm{~nm}$ range, are considered in this work after removing the atmospheric absorption bands (water vapor, $\mathrm{CO}_{2}$, etc.).

As mentioned above, a preliminary step, based on the $|\mathrm{CC}|$ calculated between the reference spectrum of the solar panels and each measured spectrum, is considered in order to select potential zones (with pixels having high $|\mathrm{CC}|$ values, i.e., higher than 0.9) that contain the considered urban material (Figure 5). Also, a visual inspection of the above full hyperspectral image is considered to check that this preprocessing step selected relevant zones. After that, three $50 \times 50$-pixel sub-images (Figure 6), containing the main materials present in the observed scene, are selected to apply the considered methods to these real airborne hyperspectral data. This selection is also performed by checking the existence of photovoltaic panels, in the considered urban region, using very high-spatial-resolution $(0.1 \mathrm{~m})$ RGB ortho-images (Figure 7 ) of the same zones.

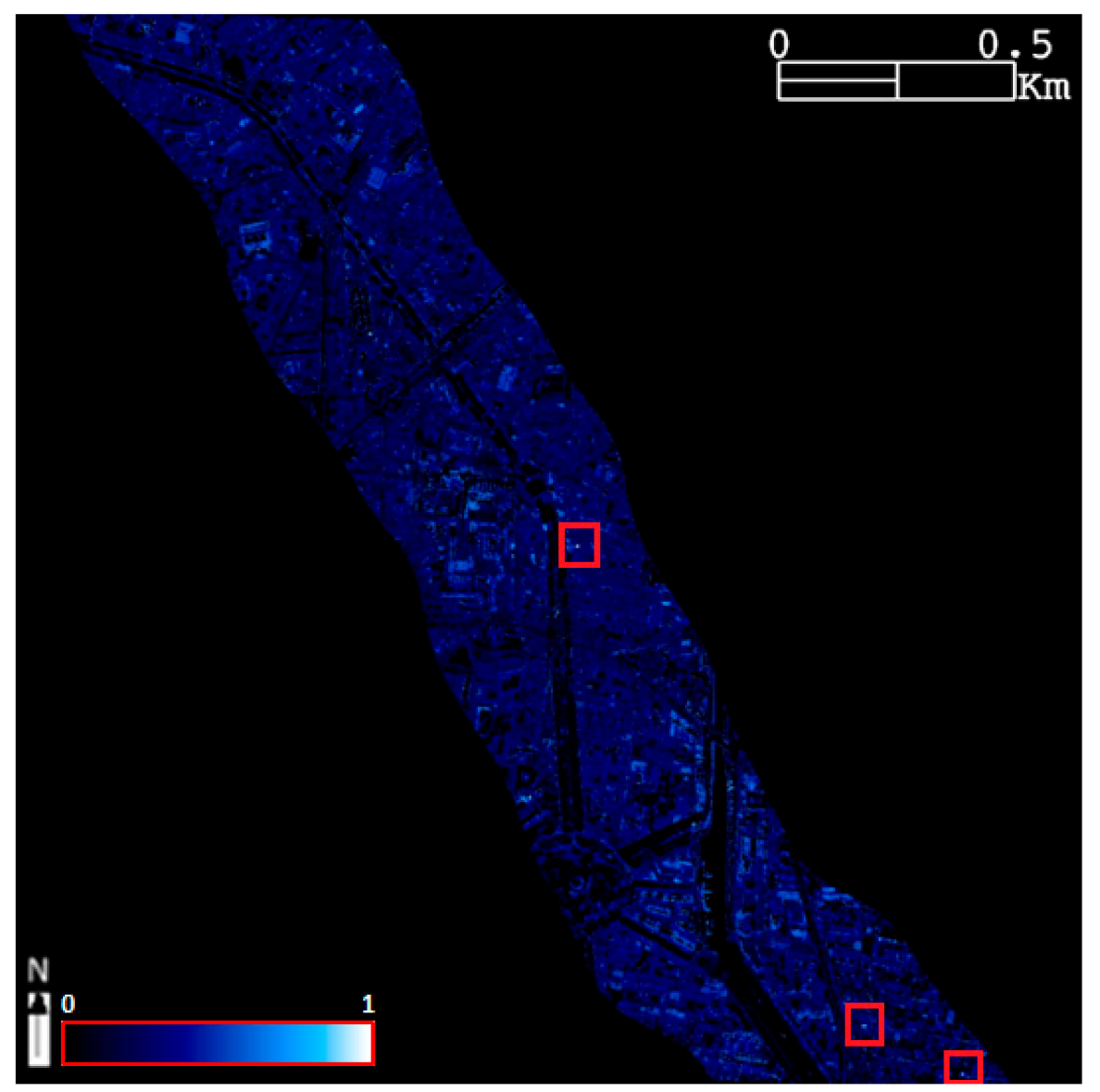

Figure 5. The obtained similarity map (based on the $|C C|$ ) of the considered full hyperspectral image. Red rectangles surround potential zones (with pixels having high $|C C|$ values). 


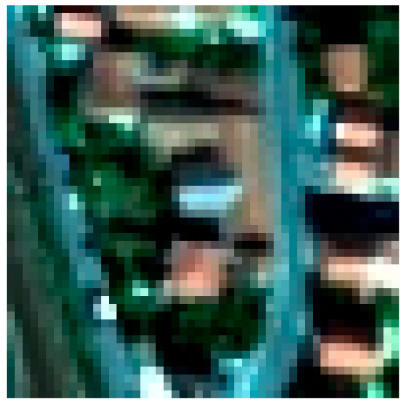

(a)

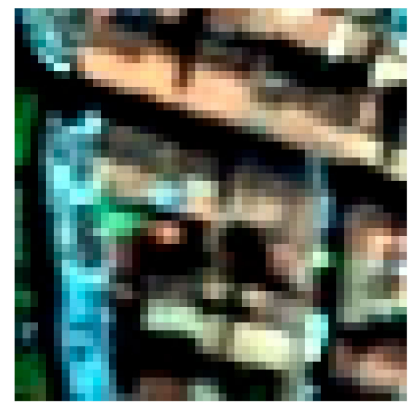

(b)

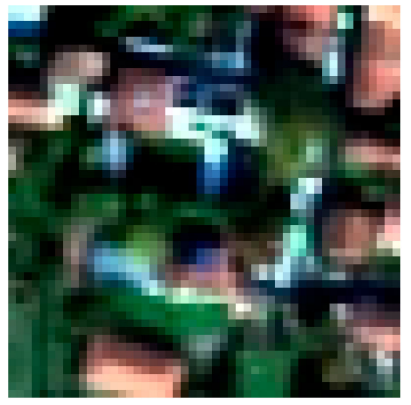

(c)

Figure 6. True color image composite of the three selected hyperspectral sub-images: (a) sub-image 1, (b) sub-image 2 , and (c) sub-image 3 .

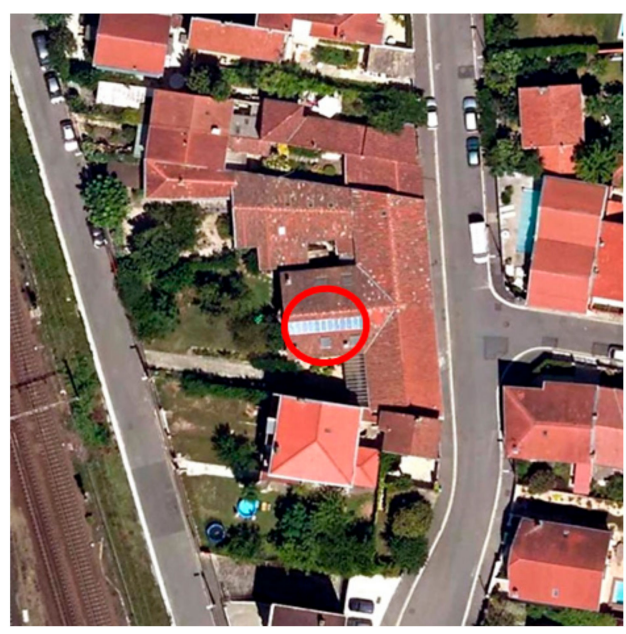

(a)

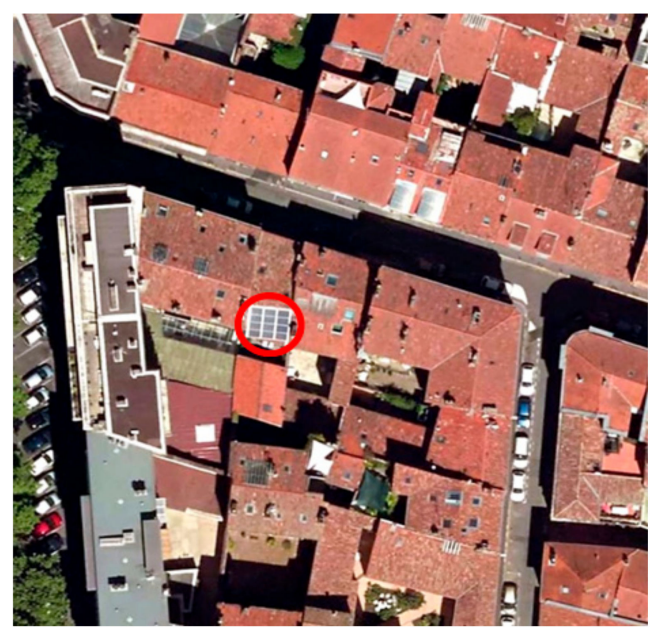

(b)

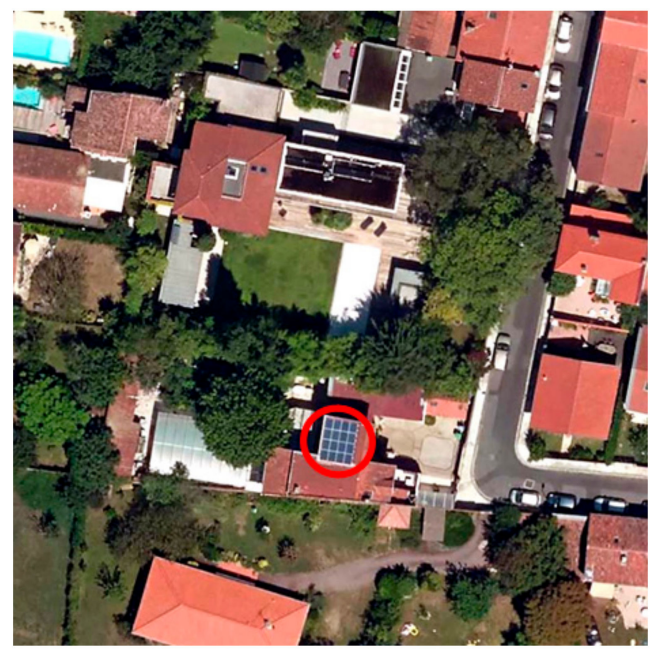

(c)

Figure 7. The three considered very high-spatial-resolution RGB ortho-images corresponding to the three selected hyperspectral sub-images: (a) ortho-image 1, (b) ortho-image 2, and (c) ortho-image 3. Red circles surround photovoltaic panels. 


\section{Experimental Results}

\subsection{Performance Evaluation Criteria}

When considering the realistic synthetic data, the Normalized Mean Square Error (NMSE) and the absolute value of the correlation coefficient $(|\mathrm{CC}|)$, calculated between the original and estimated photovoltaic panel abundance fractions, are considered to evaluate the performance of all the tested methods. These criteria are defined as

$$
\begin{gathered}
\text { NMSE }=\frac{\left\|s_{p v}-\widetilde{s}_{p v}\right\|_{F}^{2}}{\left\|s_{p v}\right\|_{F}^{2}} \\
\mathrm{CC}=\frac{\left\langle s_{p v}, \widetilde{s}_{p v}\right\rangle}{\left\|s_{p v}\right\| .\left\|\widetilde{s}_{p v}\right\|}
\end{gathered}
$$

where $\widetilde{s}_{p v}$ is the estimate of the photovoltaic panel abundance fractions map $s_{p v}$ (in one dimensional vector form).

A smaller value of the NMSE criterion corresponds to a better detection / area estimation for solar panels. Also, and when considering the $|\mathrm{CC}|$ criterion, a greater value corresponds to a better detection / area estimation for these solar systems.

When considering the real data, and in addition to a visual inspection of the provided results, estimated surfaces of solar panels are compared with those manually digitized on the very high-spatial-resolution ortho-images. As mentioned above, these estimated areas are calculated from the abundance fractions obtained by using tested NMF-unmixing-based methods, after thresholding abundance fractions to prevent several false detections and to obtain a high precision (the used threshold is empirically set to 0.3 , which gives the best results after performing tests with different threshold values between 0.2 and 0.4 with a step of 0.05 ), or from the solar panel pixels classified by using the tested one-class-classification-based approach described hereafter.

\subsection{Description of Tests}

The proposed Grd-Part-NMF (with fixed learning rate matrices set to $10^{-3}$ for each element of these matrices) and Mult-Part-NMF approaches are tested on the considered data sets. The standard gradient-based (with the same fixed learning rate matrices) and multiplicative NMF methods (Grd-NMF and Multi-NMF) $[11,24,25]$ are also considered in the experiments performed for comparison purposes. The maximum number of iterations considered in these NMF-unmixing-based methods is set to 1000 . The threshold value of the convergence criterion (20) is set to the default MATLAB epsilon value (i.e., $10^{-6}$ ) for all these used methods after running them with different threshold values (between $10^{-3}$ and $10^{-9}$ with a geometric step of $10^{-1}$, thus including the chosen value), and after noticing that the obtained performances are almost the same. Also, the VCA method is applied to estimate, as explained above, the initial unknown endmember spectra that are used in the optimization stage of these tested methods. Again, and as mentioned previously, it should be noted that the mean spectrum of the ground-measured solar panel spectra is considered to be the only known spectrum (i.e., $L_{1}=1$ ) in all of the conducted tests. Moreover, each initial value of the matrix $\widetilde{S}$ is set to $1 / L$.

For the realistic synthetic data, and since each pixel of these data is created by using one randomly selected spectrum from each the four considered sets, and in order to minimize the random effect of the results, one hundred runs (for the whole process: from the data creation to the results evaluation) are performed and the minimum ( $\mathrm{min}$ ), maximum (max), mean and standard deviation (std) values of the considered performance evaluation criteria are reported. It is clear here that for these synthetic data, $L=4$ and therefore $L_{2}=3$ (since $L_{1}=1$ ).

For the considered real data, and for the three sub-images, the estimated number of endmembers $L$ is 12 , and thus $L_{2}=11$ (again since $L_{1}=1$ ). Also, and in addition to the above tested NMF-unmixing-based 
methods (Grd-Part-NMF, Multi-Part-NMF, Grd-NMF, and Multi-NMF), a one-class-classification-based method is applied to these real data. This approach contains two stages. In the first one, the correlation coefficient is calculated between each observed pixel-spectrum and the known mean of ground-measured photovoltaic panel spectra. In the second stage, only pixels with correlation coefficients higher than a fixed threshold (empirically set to 0.9 that gives the best performances after performing tests with three threshold values: $0.85,0.9$ and 0.95 ) are considered (again to avoid false detections and to obtain a high precision) to extract photovoltaic panels.

\subsection{Results}

The following tables (Tables 1 and 2) show the values of the performance criteria obtained for the realistic synthetic data.

In the following figures (Figures 8 and 9), histograms of the considered performance criteria (for the realistic synthetic data) are given.

Table 1. Photovoltaic panel NMSE values (\%).

\begin{tabular}{ccccc}
\hline & Grd-Part-NMF & Multi-Part-NMF & Grd-NMF & Multi-NMF \\
\hline Min & 69.21 & $\mathbf{1 1 . 4 6}$ & 89.84 & 85.26 \\
Max & 112.46 & $\mathbf{4 4 . 1 8}$ & 115.81 & 109.82 \\
Mean & 84.80 & $\mathbf{2 3 . 7 3}$ & 103.03 & 98.64 \\
Std & 07.77 & 08.72 & 06.57 & $\mathbf{0 6 . 5 1}$ \\
\hline
\end{tabular}

Table 2. Photovoltaic panel $|C C|$ values.

\begin{tabular}{ccccc}
\hline & Grd-Part-NMF & Multi-Part-NMF & Grd-NMF & Multi-NMF \\
\hline Min & 0.56 & 0.94 & 0.35 & 0.37 \\
Max & 0.84 & 0.99 & 0.59 & 0.61 \\
Mean & 0.74 & 0.98 & 0.47 & 0.49 \\
Std & 0.05 & 0.01 & 0.65 & 0.07 \\
\hline
\end{tabular}

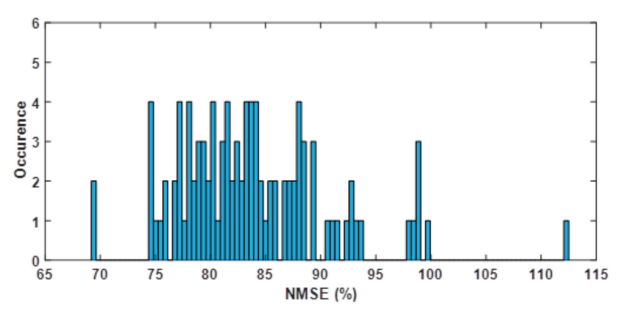

(a)

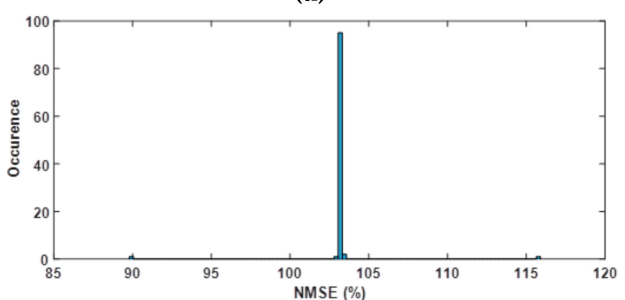

(c)

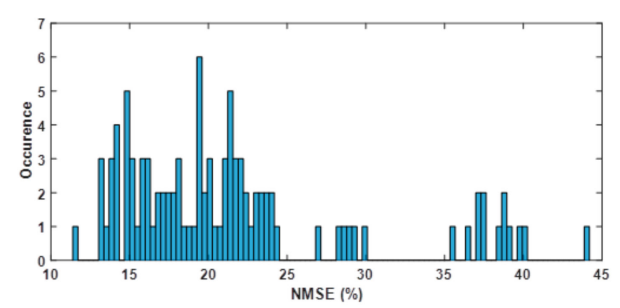

(b)

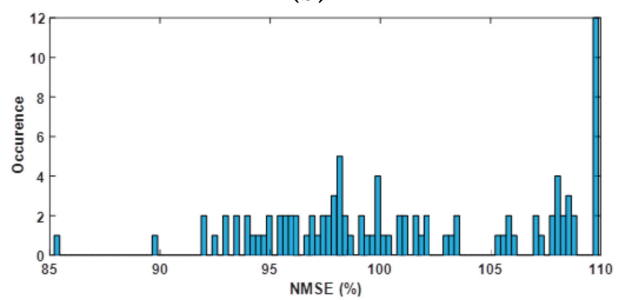

(d)

Figure 8. Histograms of the NMSE criterion values (\%) obtained by the tested methods: (a) Grd-Part-NMF, (b) Multi-Part-NMF, (c) Grd-NMF and (d) Multi-NMF. 


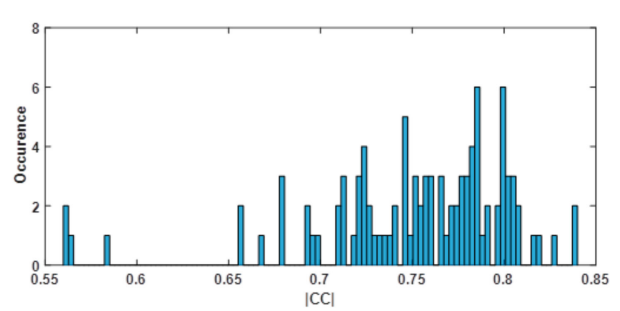

(a)

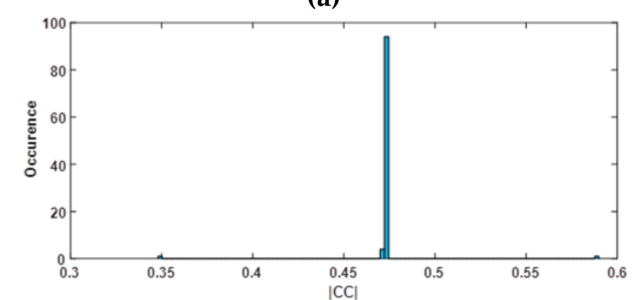

(c)

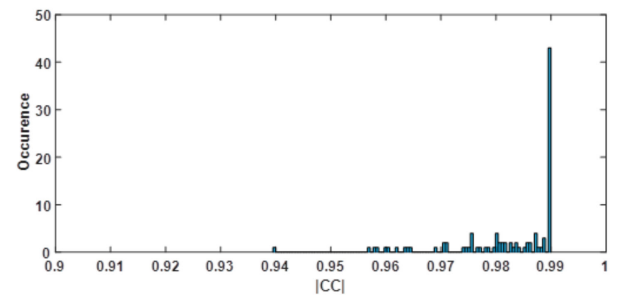

(b)

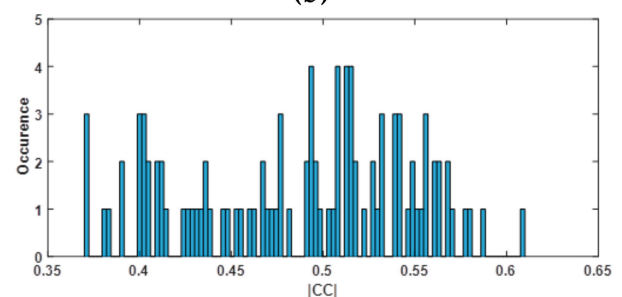

(d)

Figure 9. Histograms of the $|C C|$ criterion values obtained by the tested methods: (a) Grd-Part-NMF, (b) Multi-Part-NMF, (c) Grd-NMF and (d) Multi-NMF.

The following figures (Figures 10-12) show the estimated (by the tested NMF-unmixing-based algorithms) photovoltaic panel abundance fraction maps for the three considered real airborne hyperspectral sub-images. These figures also show the obtained one-class (solar panels) classification maps. 


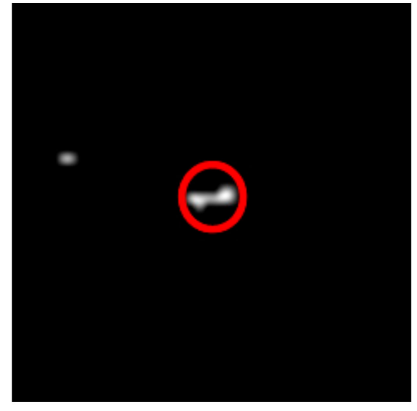

(a)

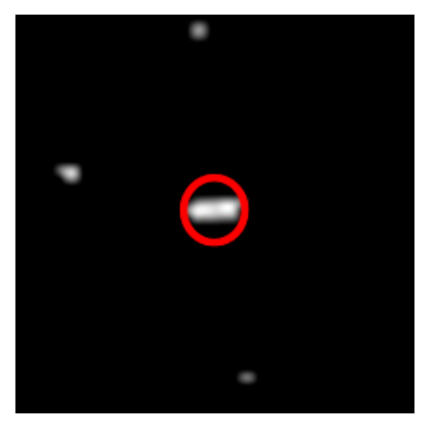

(c)

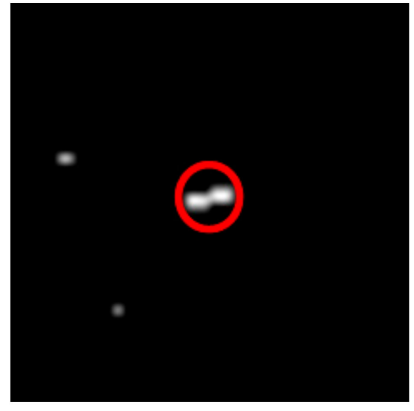

(b)

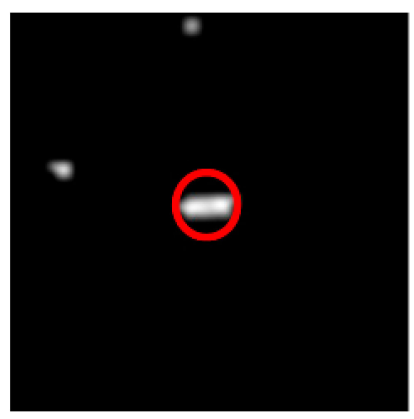

(d)

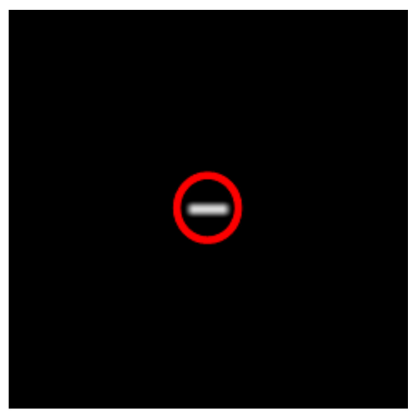

(e)

Figure 10. The obtained photovoltaic panel abundance fraction and classification maps for the real sub-image 1: (a) Grd-Part-NMF, (b) Multi-Part-NMF, (c) Grd-NMF, (d) Multi-NMF and (e) one-class-classification-based. Red circles surround photovoltaic panels. 


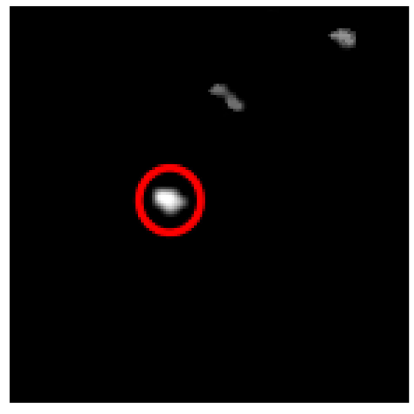

(a)

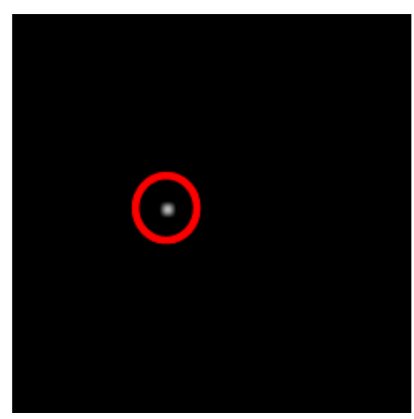

(c)

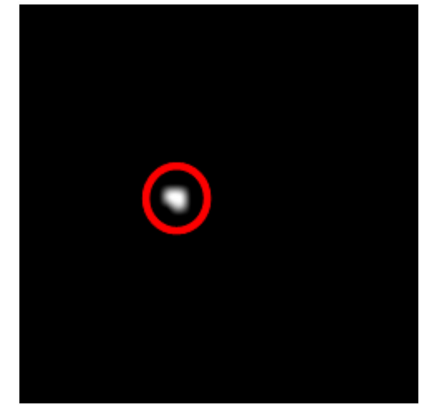

(b)

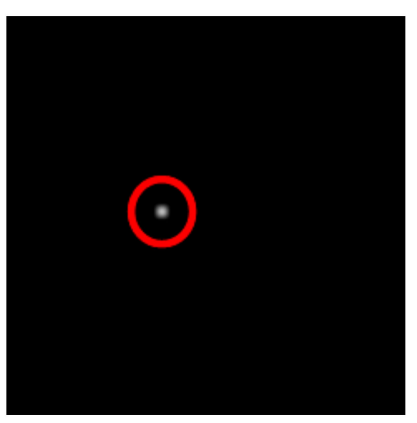

(d)

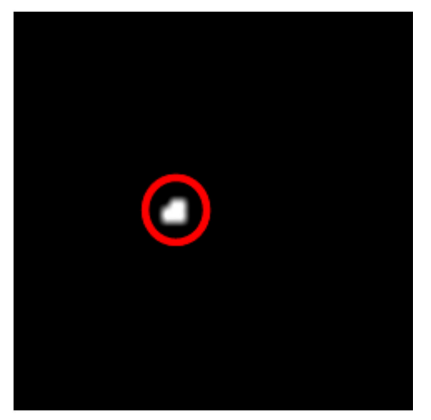

(e)

Figure 11. The obtained photovoltaic panel abundance fraction and classification maps for the real sub-image 2: (a) Grd-Part-NMF, (b) Multi-Part-NMF, (c) Grd-NMF, (d) Multi-NMF and (e) one-class-classification-based. Red circles surround photovoltaic panels. 


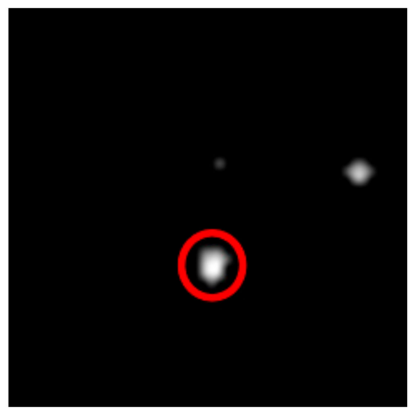

(a)

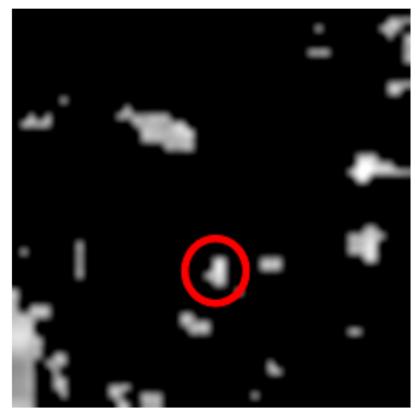

(c)

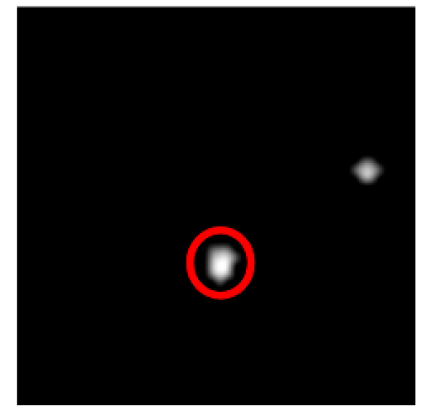

(b)

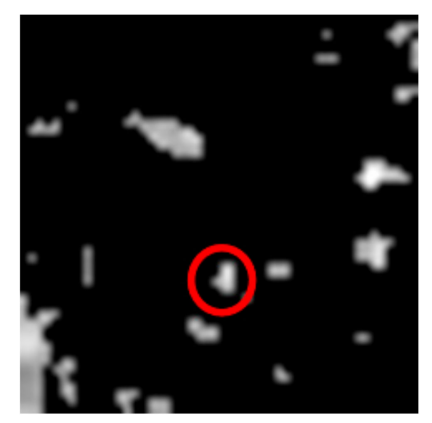

(d)

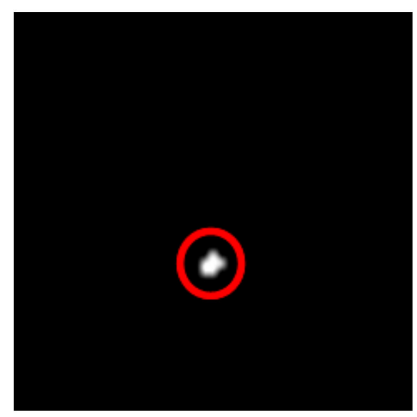

(e)

Figure 12. The obtained photovoltaic panel abundance fraction and classification maps for the real sub-image 3: (a) Grd-Part-NMF, (b) Multi-Part-NMF, (c) Grd-NMF, (d) Multi-NMF and (e) one-class-classification-based. Red circles surround photovoltaic panels.

Table 3 below shows the photovoltaic panel areas obtained from the three real hyperspectral sub-images.

Table 3. Photovoltaic panel areas $\left(\mathrm{m}^{2}\right)$ for: (a) sub-image 1, (b) sub-image 2, and (c) sub-image 3.

\begin{tabular}{ccccccc}
\hline & $\begin{array}{c}\text { Manual } \\
\text { Digitization }\end{array}$ & Grd-Part-NMF & Multi-Part-NMF & Grd-NMF & Multi-NMF & Classification \\
\hline (a) & 15 & 14.57 & 15.27 & 22.27 & 21.26 & $12.80(5$ pixels $)$ \\
(b) & 12 & 13.33 & 13.06 & 01.10 & 01.10 & $20.48(8$ pixels $)$ \\
(c) & 16 & 16.20 & 15.65 & 90.58 & 88.28 & $15.36(6$ pixels $)$ \\
\hline
\end{tabular}




\subsection{Computational Complexity and Run Time}

On the one hand, the proposed (and also the tested literature NMF-unmixing-based) approaches are very easy to implement, and their core (i.e., their update rules) contains only simple and direct matrix operations: standard matrix addition and product, element-wise matrix product and division, and matrix transposition. These matrix operations make the algorithmic complexity of the proposed (and the tested literature NMF-based) approaches very limited.

On the other hand, the running time of the core of tested NMF-unmixing-based methods depends on the input image size, the number of materials in the observed scene, the maximum number of iterations and the stopping criterion (20): based on the above description of tests, the running time of these methods (by using an Intel(R) Core(TM) i5 processor running at $2.40 \mathrm{GHz}$ and a memory capacity of $8 \mathrm{~GB}$ ) is about one second for each run when considering realistic synthetic data, and is less than $20 \mathrm{~s}$ for each considered real sub-image.

\section{Discussion}

For the realistic synthetic data, Tables 1 and 2, and Figures 8 and 9, globally show that the designed approaches achieve much better detection of solar panels than the tested literature NMF-unmixing-based algorithms. Indeed, these tables reveal that the designed Multi-Part-NMF (respectively Grd-Part-NMF) algorithm is able to reach $23.73 \%$ (respectively $84.80 \%$ ) mean NMSE for solar panels, whereas the literature Multi-NMF (respectively Grd-NMF) technique attains a mean of 98.64\% (respectively $103.03 \%$ ). In the same way, and in terms of the $|\mathrm{CC}|$ criterion, the proposed Multi-Part-NMF (respectively Grd-Part-NMF) algorithm reaches a mean of 0.98 (respectively 0.74), whereas the literature Multi-NMF (respectively Grd-NMF) method attains a mean of 0.49 (respectively 0.47). Also, the above tables show that the other calculated NMSE and $|C C|$ values (minimum, maximum and standard deviation) follow, globally, the same trends as the mean values.

For the considered real data, Figures 10-12 confirm, by visual checking with ortho-images, that the designed algorithms are able to better detect all solar panels than the tested standard techniques. Also, Table 3 shows that the designed algorithms are able to automatically estimate solar panel surfaces much more precisely than the tested standard methods. Indeed, the areas obtained with the proposed methods, for the three considered sub-images, are much closer to manually digitized areas than those obtained by the other tested methods from the literature. For sub-image 1, with a manually obtained area of $15 \mathrm{~m}^{2}$ of photovoltaic panels, the proposed Multi-Part-NMF (respectively Grd-Part-NMF) method gives an area of $15.27 \mathrm{~m}^{2}$ (respectively $14.57 \mathrm{~m}^{2}$ ), whereas the standard Multi-NMF (respectively Grd-NMF) method estimates an area of $21.26 \mathrm{~m}^{2}$ (respectively $22.27 \mathrm{~m}^{2}$ ). Moreover, 5 pixels (corresponding to an area of $12.80 \mathrm{~m}^{2}$ ) are extracted by the considered one-class-classification-based approach. For the second sub-image, which contains an area, again manually digitized, of $12 \mathrm{~m}^{2}$ of solar panels, the two proposed methods give areas of around $13 \mathrm{~m}^{2}$, whereas the literature NMF-unmixing-based approaches estimate areas of around $1 \mathrm{~m}^{2}$. Also, an area of $20.48 \mathrm{~m}^{2}$ (corresponding to 8 pixels) is estimated by the tested one-class-classification-based method. For sub-image 3, with an area of $16 \mathrm{~m}^{2}$ of panels, manually calculated from the corresponding ortho-image, the proposed Grd-Part-NMF (respectively Multi-Part-NMF) method estimates an area of $16.20 \mathrm{~m}^{2}$ (respectively $15.65 \mathrm{~m}^{2}$ ). The two tested standard NMF-unmixing-based methods give areas of about $90 \mathrm{~m}^{2}$ and the one-class-classification-based method extracts 6 pixels (corresponding to an area of $15.36 \mathrm{~m}^{2}$ ) of photovoltaic panels.

In general, and from all obtained results, it is clear that the proposed methods achieve the desired goal of automatically detecting photovoltaic panels and estimating their areas correctly, and more precisely than the tested standard methods. For the tested literature NMF-unmixing-based methods, this result is expected, since, and unlike the proposed methods, they update all variables, including the panel spectrum that is supposed to be known. This update of the panel spectrum may have a negative influence on the abundance fraction estimation of this material, which may explain the obtained results. Also, and for the considered one-class-classification-based method, which gives acceptable but poorer results than those obtained by the proposed methods, the observation is the same: this result is 
expected since this literature method, and unlike the proposed methods, considers the total occupied area per pixel containing photovoltaic panels.

\section{Conclusions}

In these investigations, two approaches, called Grd-Part-NMF and Mult-Part-NMF, were designed to automatically detect photovoltaic panels in urban hyperspectral remote sensing data and to estimate their surfaces. These original approaches, related to LSU techniques, are based on NMF. The designed methods, which use known photovoltaic panel spectra, optimize a criterion with new designed iterative update rules. The first approach uses an iterative projected gradient descent algorithm, whereas in the second one, an iterative multiplicative gradient-based algorithm was designed.

The proposed methods, which are very easy to implement, were tested on realistic synthetic and real airborne hyperspectral data, and their efficiency was evaluated with commonly used performance criteria when considering the synthetic data. For the real data, the performance of the designed methods was evaluated by comparing obtained photovoltaic panel areas with those calculated by a manual digitization on ortho-images of the same regions with very high spatial resolution.

The obtained results show that the proposed methods yield very satisfactory results and prove to be very attractive in the framework of the photovoltaic panel detection in urban hyperspectral remote sensing data, and can be of great help to electricity grid operators and decision makers. Also, according to the obtained results, the proposed methods significantly outperform, when considering the synthetic data, the tested NMF-unmixing-based approaches from the literature. In addition, still for the synthetic data, the proposed Multi-Part-NMF method gives the best results in comparison with the proposed Grd-Part-NMF method. When considering the real data, and in addition to their superiority in comparison with these literature methods, they also surpass the tested standard one-class-classification-based approach. Moreover, and still considering the real data, the two proposed methods give comparable results with, globally, a slight advantage for the Multi-Part-NMF method.

An interesting extension of this work may consist in developing new measures or adapting the standard $F$-score measure to assess in more detail the performance of the proposed approaches, by evaluating false positive/negative in the detection process. Indeed, the standard F-score measure is used when the detection is binary-valued, which is not the case for the proposed methods.

Other potential extensions of these investigations will mainly consist in applying the proposed approaches to automatically detect and estimate surfaces of other materials in urban hyperspectral remote sensing data, such as solar water heaters and roof windows (and in analyzing if it is possible to distinguish them from the solar panels), and also surfaces of some impervious surfaces such as asphalt, which contributes to the increase of the heat islands phenomenon in urban regions. Moreover, it would be interesting to apply the proposed methods to detect and estimate areas of non-urban materials in hyperspectral data, such as ground surfaces potentially indicating the presence of rocks containing native or complex metallic minerals.

Author Contributions: Data curation, T.H. and A.L.B.; Formal analysis, X.B.; Funding acquisition, C.W.; Investigation, M.S.K., F.Z.B. and Y.D.; Methodology, M.S.K., F.Z.B., Y.D. and K.D.; Project administration, C.W.; Software, M.S.K., F.Z.B. and K.D.; Supervision, C.W.; Writing—original draft, M.S.K.; Writing—review \& editing, M.S.K., Y.D., X.B. and C.W.

Funding: This research was funded by the French ANR project on HYperspectral imagery for Environmental urban Planning (HYEP, no. ANR 14-CE22-0016-01).

Conflicts of Interest: The authors declare no conflict of interest.

\section{References}

1. Malof, J.M.; Bradbury, K.; Collins, L.M.; Newell, R.G. Automatic detection of solar photovoltaic arrays in high resolution aerial imagery. Appl. Energy 2016, 183, 229-240. [CrossRef]

2. Alam, M.J.E.; Muttaqi, K.M.; Sutanto, D. An approach for online assessment of rooftop solar PV impacts on low-voltage distribution networks. IEEE Trans. Sustain. Energy 2014, 5, 663-672. [CrossRef] 
3. Chersin, A.; Ongsakul, W.; Mitra, J.; Member, S. Improving of uncertain power generation of rooftop solar PV using battery storage. In Proceedings of the 2014 International Conference and Utility Exhibition on Green Energy for Sustainable Development (ICUE), Pattaya, Thailand, 19-21 March 2014.

4. Puttemans, S.; van Ranst, W.; Goedeme, T.; Kerle, N.; Gerke, M.; Lefevre, S. Detection of photovoltaic installations in RGB aerial imaging: A comparative study. In GEOBIA 2016: Solutions and Synergies; University of Twente: Enschede, The Netherlands, 14-16 September 2016.

5. Chang, C.-I. Hyperspectral Data Processing: Algorithm Design and Analysis; John Wiley \& Sons: Hoboken, NJ, USA, 2013.

6. Czirjak, D.W. Detecting photovoltaic solar panels using hyperspectral imagery and estimating solar power production. J. Appl. Rem. Sens. 2017, 11, 026007. [CrossRef]

7. Karoui, M.S.; Benhalouche, F.Z.; Deville, Y.; Djerriri, K.; Briottet, X.; Le Bris, A. Detection and Area Estimation for Photovoltaic Panels in Urban Hyperspectral Remote Sensing Data by An Original Nmf-Based Unmixing Method. In Proceedings of the IGARSS 2018-2018 IEEE International Geoscience and Remote Sensing Symposium, Valencia, Spain, 22-27 July 2018; pp. 1640-1643.

8. Deville, Y. Blind Source Separation and Blind Mixture Identification Methods. In Wiley Encyclopedia of Electrical and Electronics Engineering; John Wiley \& Sons, Inc.: Hoboken, NJ, USA, 2016; pp. 1-33.

9. Comon, P.; Jutten, C. Handbook of Blind Source Separation: Independent Component Analysis and Applications; Academic Press: Oxford, UK, 2010.

10. Bioucas-Dias, J.M.; Plaza, A.; Dobigeon, N.; Parente, M.; Du, Q.; Gader, P.; Chanussot, J. Hyperspectral Unmixing Overview: Geometrical, Statistical, and Sparse Regression-Based Approaches. IEEE J. Sel. Top. Appl. Earth Obs. Remote Sens. 2012, 5, 354-379. [CrossRef]

11. Cichocki, A.; Zdunek, R.; Phan, A.H.; Amari, S.-I. Nonnegative Matrix and Tensor Factorizations; ohn Wiley \& Sons, Inc.: Hoboken, NJ, USA, 2009.

12. Dobigeon, N.; Tourneret, J.-Y.; Richard, C.; Bermudez, J.C.M.; McLaughlin, S.; Hero, A.O. Nonlinear Unmixing of Hyperspectral Images: Models and Algorithms. IEEE Signal Process. Mag. 2014, 31, 82-94. [CrossRef]

13. Meganem, I.; Deliot, P.; Briottet, X.; Deville, Y.; Hosseini, S. Linear-Quadratic Mixing Model for Reflectances in Urban Environments. IEEE Trans. Geosci. Remote Sens. 2014, 52, 544-558. [CrossRef]

14. Deville, Y.; Duarte, L.T. An Overview of Blind Source Separation Methods for Linear-Quadratic and Post-nonlinear Mixtures. Inf. Technol. Bio-Med Inform. 2015, 9237, 155-167.

15. Deville, Y. Matrix factorization for bilinear blind source separation: Methods, separability and conditioning. In Proceedings of the 2015 23rd European Signal Processing Conference (EUSIPCO), Nice, France, 31 August-4 September 2015; pp. 1900-1904.

16. Meganem, I.; Deville, Y.; Hosseini, S.; Deliot, P.; Briottet, X. Linear-Quadratic Blind Source Separation Using NMF to Unmix Urban Hyperspectral Images. IEEE Trans. Signal Process. 2014, 62, 1822-1833. [CrossRef]

17. Benhalouche, F.Z.; Deville, Y.; Karoui, M.S.; Ouamri, A. Bilinear matrix factorization using a gradient method for hyperspectral endmember spectra extraction. In Proceedings of the 2016 IEEE International Geoscience and Remote Sensing Symposium (IGARSS), Beijing, China, 10-15 July 2016; pp. 6565-6568.

18. Benhalouche, F.Z.; Deville, Y.; Karoui, M.S.; Ouamri, A. Hyperspectral endmember spectra extraction based on constrained linear-quadratic matrix factorization using a projected gradient method. In Proceedings of the 2016 IEEE 26th International Workshop on Machine Learning for Signal Processing (MLSP), Vietri sul Mare, Italy, 13-16 September 2016; pp. 1-6.

19. Limem, A.; Delmaire, G.; Puigt, M.; Roussel, G.; Courcot, D. Non-negative Matrix Factorization using weighted beta divergence and equality constraints for industrial source apportionment. In Proceedings of the 2013 IEEE International Workshop on Machine Learning for Signal Processing (MLSP), Southampton, UK, 22-25 September 2013; pp. 1-6.

20. Tong, L.; Zhou, J.; Qian, Y.; Bai, X.; Gao, Y. Nonnegative-Matrix-Factorization-Based Hyperspectral Unmixing With Partially Known Endmembers. IEEE Trans. Geosci. Remote Sens. 2016, 54, 1-14. [CrossRef]

21. Karoui, M.S.; Deville, Y.; Hosseini, S.; Ouamri, A.; Hosseini, S. A new spatial sparsity-based method for extracting endmember spectra from hyperspectral data with some pure pixels. In Proceedings of the 2012 IEEE International Geoscience and Remote Sensing Symposium, Munich, Germany, 22-27 July 2012; pp. 3074-3077. 
22. Karoui, M.S.; Deville, Y.; Hosseini, S.; Ouamri, A. Blind spatial unmixing of multispectral images: New methods combining sparse component analysis, clustering and non-negativity constraints. Pattern Recognit. 2012, 45, 4263-4278. [CrossRef]

23. Karoui, M.S.; Deville, Y.; Hosseini, S.; Ouamri, A.; Hosseini, S. Blind unmixing of remote sensing data with some pure pixels: Extension and comparison of spatial methods exploiting sparsity and nonnegativity properties. In Proceedings of the 2013 8th International Workshop on Systems, Signal Processing and their Applications (WoSSPA), Algiers, Algeria, 12-15 May 2013; pp. 42-49.

24. Lee, D.D.; Seung, H.S. Learning the parts of objects by non-negative matrix factorization. Nature 1999, 401, 788-791. [CrossRef] [PubMed]

25. Lee, D.D.; Seung, H.S. Algorithms for non-negative matrix factorization. In Advances in Neural Information Processing Systems 13, Proceedings of the 2000 Conference, Denver, CO, USA, 27-30 November 2000; Leen, T.K., Dietterich, T.G., Tresp, V., Eds.; MIT Press: Cambridge, MA, USA, 2001; pp. 556-562.

26. Lin, C.-J. Projected Gradient Methods for Nonnegative Matrix Factorization. Neural Comput. 2007, 19, 2756-2779. [CrossRef] [PubMed]

27. Petersen, K.B.; Pedersen, M.S. The Matrix Cookbook; Technical University Denmark: Lyngby, Denmark, 2008.

28. Nascimento, J.M.P.; Dias, J.M.B. Vertex component analysis: A fast algorithm to unmix hyperspectral data. IEEE Trans. Geosci. Remote Sens. 2005, 43, 898-910. [CrossRef]

29. Bioucas-Dias, J.M. A variable splitting augmented Lagrangian approach to linear spectral unmixing. In Proceedings of the 2009 First Workshop on Hyperspectral Image and Signal Processing: Evolution in Remote Sensing, Grenoble, France, 26-28 August 2009; pp. 1-4.

30. Bioucas-Dias, J.; Nascimento, J. Hyperspectral Subspace Identification. IEEE Trans. Geosci. Remote Sens. 2008, 46, 2435-2445. [CrossRef]

31. Heinz, D.C.; Chang, C.I. Fully constrained least squares linear spectral mixture analysis method for material quantification in hyperspectral imagery. IEEE Trans. Geosci. Remote Sens. 2001, 39, 529-545. [CrossRef]

32. Revel, C.; Deville, Y.; Achard, V.; Briottet, X.; Weber, C. Inertia-Constrained Pixel-by-Pixel Nonnegative Matrix Factorisation: A Hyperspectral Unmixing Method Dealing with Intra-Class Variability. Remote Sens. 2018, 10, 1706. [CrossRef]

33. Brabant, C.; Alvarez-Vanhard, E.; Laribi, A.; Morin, G.; Nguyen, K.T.; Thomas, A.; Houet, T.; Vanhard, A. Comparison of Hyperspectral Techniques for Urban Tree Diversity Classification. Remote Sens. 2019, 11, 1269. [CrossRef]

34. Adeline, K.; Le Bris, A.; Coubard, F.; Briottet, X.; Paparoditis, N.; Viallefont, F.; Riviere, N.; Papelard, J.-P.; David, N.; Déliot, P.; et al. Description de la campagne aéroportée UMBRA: Étude de l'impact anthropique sur les écosystèmes urbains et naturels avec des images THR multispectrales et hyperspectrales. Revue Française de Photogrammétrie et de Télédétection 2013, 202, 79-92.

35. Miesch, C.; Poutier, L.; Achard, V.; Briottet, X.; Lenot, X.; Boucher, Y. Direct and inverse radiative transfer solutions for visible and near-infrared hyperspectral imagery. IEEE Trans. Geosci. Remote Sens. 2005, 43, 1552-1562. [CrossRef]

(C) 2019 by the authors. Licensee MDPI, Basel, Switzerland. This article is an open access article distributed under the terms and conditions of the Creative Commons Attribution (CC BY) license (http://creativecommons.org/licenses/by/4.0/). 